\title{
Integral theorems for the quaternionic $G$-monogenic mappings
}

\author{
V.S. Shpakivskyi and T.S. Kuzmenko
}

\begin{abstract}
In the paper [1] considered a new class of quaternionic mappings, socalled $G$-monogenic mappings. In this paper we prove analogues of classical integral theorems of the holomorphic function theory: the Cauchy integral theorems for surface and curvilinear integrals, and the Cauchy integral formula for $G$-monogenic mappings.
\end{abstract}

Introduction. The Cauchy integral theorem and Cauchy integral formula for holomorphic functions of the complex variable are fundamental results of the classical complex analysis. Analogues of these results are also important tools in the quaternionic analysis.

Maybe the first quaternionic analogues of the mentioned results for a surface in $\mathbb{R}^{3}$ are obtained by G. Moisil and N. Theodoresco [2]. Namely, they proved some analogues of the Cauchy's theorems for a smooth surface and for continuously differentiable functions $f$ satisfying the equality

$$
\frac{\partial f}{\partial x} i+\frac{\partial f}{\partial y} j+\frac{\partial f}{\partial z} k=0
$$

where $i, j, k$ are the basis quaternionic units.

Key Words: quaternion algebra, $G$-monogenic mapping, Cauchy integral theorem, Cauchy integral formula.

2010 Mathematics Subject Classification: Primary 30G35; Secondary 11R52.

Received: 17.12.2014

Revised: 05.01 .2015

Accepted: 07.01.2015 
R. Fueter [3] transferred the results of paper [2] for a smooth surface in $\mathbb{R}^{4}$ and for so-called the regular functions $f$ which by definition satisfies the equality

$$
\frac{\partial f}{\partial t}+\frac{\partial f}{\partial x} i+\frac{\partial f}{\partial y} j+\frac{\partial f}{\partial z} k=0 .
$$

The proofs of [2] and [3] are based on the Stokes formula.

B. Schuler [4] generalized the results of Fueter by adapting of Goursat's proof of the Cauchy's theorem. Due to this, he replaced the condition of continuity of partial derivatives to the differentiability of the real-valued components in the Stolz sense.

A. Sudbery [5] proved the Cauchy theorem and Cauchy formula under more general conditions on a function and a surface. He assumed as in the complex analysis only differentiability of real-valued components of the integrand. $\mathrm{He}$ also considered the so-called differentiable surface and the rectifiable 3 -chain.

O. Herus [6] proved the quaternionic Cauchy theorem for a surface in $\mathbb{R}^{3}$ under the same conditions on the function as in [5], but for another class of surfaces.

Some analogues of the quaternionic Cauchy theorem and Cauchy integral formula in the theory of $s$-regular functions are established in [7].

In this paper for quaternionic G-monogenic mappings we prove analogues of the Cauchy theorem for surface and curvilinear integrals and the Cauchy integral formula curvilinear integral.

\section{The algebra of complex quaternion $\mathbb{H}(\mathbb{C})$ and $G$-monogenic} mappings.

Let $\mathbb{H}(\mathbb{C})$ be the quaternion algebra over the field of complex numbers $\mathbb{C}$, whose basis consists of the unit 1 of the algebra and of the elements $I, J, K$ satisfying the multiplication rules:

$$
\begin{gathered}
I^{2}=J^{2}=K^{2}=-1, \\
I J=-J I=K, \quad J K=-K J=I, \quad K I=-I K=J .
\end{gathered}
$$

In the algebra $\mathbb{H}(\mathbb{C})$ there exists another basis $\left\{e_{1}, e_{2}, e_{3}, e_{4}\right\}$ :

$$
e_{1}=\frac{1}{2}(1+i I), \quad e_{2}=\frac{1}{2}(1-i I), \quad e_{3}=\frac{1}{2}(i J-K), \quad e_{4}=\frac{1}{2}(i J+K),
$$

where $i$ is the complex imaginary unit. Multiplication table in a new basis can be represented as

\begin{tabular}{c||c|c|c|c|}
$\cdot$ & $e_{1}$ & $e_{2}$ & $e_{3}$ & $e_{4}$ \\
\hline \hline$e_{1}$ & $e_{1}$ & 0 & $e_{3}$ & 0 \\
\hline$e_{2}$ & 0 & $e_{2}$ & 0 & $e_{4}$ \\
\hline$e_{3}$ & 0 & $e_{3}$ & 0 & $e_{1}$ \\
\hline$e_{4}$ & $e_{4}$ & 0 & $e_{2}$ & 0 \\
\hline
\end{tabular}


The unit 1 can be decomposed as $1=e_{1}+e_{2}$.

Consider linear functionals $f_{1}: \mathbb{H}(\mathbb{C}) \rightarrow \mathbb{C}$ and $f_{2}: \mathbb{H}(\mathbb{C}) \rightarrow \mathbb{C}$ satisfying the equalities

$$
\begin{array}{ll}
f_{1}\left(e_{1}\right)=f_{1}\left(e_{3}\right)=1, & f_{1}\left(e_{2}\right)=f_{1}\left(e_{4}\right)=0, \\
f_{2}\left(e_{2}\right)=f_{2}\left(e_{4}\right)=1, & f_{2}\left(e_{1}\right)=f_{2}\left(e_{3}\right)=0 .
\end{array}
$$

Let us consider the vectors

$$
i_{1}=1=e_{1}+e_{2}, \quad i_{2}=a_{1} e_{1}+a_{2} e_{2}, \quad i_{3}=b_{1} e_{1}+b_{2} e_{2},
$$

$a_{k}, b_{k} \in \mathbb{C}, k=1,2$, which are a linearly independent over the field of real numbers $\mathbb{R}$ (see $[8$, p. 223]). It means that the equality

$$
\alpha_{1} i_{1}+\alpha_{2} i_{2}+\alpha_{3} i_{3}=0, \quad \alpha_{1}, \alpha_{2}, \alpha_{3} \in \mathbb{R}
$$

holds if and only if $\alpha_{1}=\alpha_{2}=\alpha_{3}=0$.

In the algebra $\mathbb{H}(\mathbb{C})$ consider the linear span $E_{3}:=\left\{\zeta=x i_{1}+y i_{2}+\right.$ $\left.z i_{3}: x, y, z \in \mathbb{R}\right\}$ generated by the vectors $i_{1}, i_{2}, i_{3}$ over the field $\mathbb{R}$. Denote $f_{k}\left(E_{3}\right):=\left\{f_{k}(\zeta): \zeta \in E_{3}\right\}, k=1,2$. In what follows, we make the following essential assumption: $f_{1}\left(E_{3}\right)=f_{2}\left(E_{3}\right)=\mathbb{C}$. Obviously, it holds if and only if at least one of the numbers in each of pairs $\left(a_{1}, b_{1}\right)$ or $\left(a_{2}, b_{2}\right)$ belongs to $\mathbb{C} \backslash \mathbb{R}$.

Let us introduce the notations

$$
\xi_{1}:=f_{1}(\zeta)=x+y a_{1}+z b_{1}, \quad \xi_{2}:=f_{2}(\zeta)=x+y a_{2}+z b_{2} .
$$

Now, the element $\zeta \in E_{3}$ can be represented in the form $\zeta=\xi_{1} e_{1}+\xi_{2} e_{2}$.

A set $S \subset \mathbb{R}^{3}$ is associated with the set $S_{\zeta}:=\left\{\zeta=x i_{1}+y i_{2}+z i_{3}\right.$ : $(x, y, z) \in S\}$ in $E_{3}$. We also note that a topological property of a set $S_{\zeta}$ in $E_{3}$ understand as the same topological property of the set $S$ in $\mathbb{R}^{3}$. For example, we will say that a curve $\gamma_{\zeta} \subset E_{3}$ is homotopic to the zero if $\gamma \subset \mathbb{R}^{3}$ is homotopic to the zero, etc. Let $\Omega_{\zeta}$ be a domain in $E_{3}$.

A continuous mapping $\Phi: \Omega_{\zeta} \rightarrow \mathbb{H}(\mathbb{C})\left(\right.$ or $\left.\widehat{\Phi}: \Omega_{\zeta} \rightarrow \mathbb{H}(\mathbb{C})\right)$ is right-Gmonogenic (or resp. left-G-monogenic) in a domain $\Omega_{\zeta} \subset E_{3}$, if $\Phi$ (or resp. $\widehat{\Phi})$ is differentiable in the sense of the Gateaux in every point of $\Omega_{\zeta}$, i. e. if for every $\zeta \in \Omega_{\zeta}$ there exists an element $\Phi^{\prime}(\zeta) \in \mathbb{H}(\mathbb{C})\left(\right.$ or resp. $\widehat{\Phi}^{\prime}(\zeta) \in \mathbb{H}(\mathbb{C})$ ) such that

$$
\begin{gathered}
\lim _{\varepsilon \rightarrow 0+0}(\Phi(\zeta+\varepsilon h)-\Phi(\zeta)) \varepsilon^{-1}=h \Phi^{\prime}(\zeta) \quad \forall h \in E_{3} \\
\left(\text { or resp. } \lim _{\varepsilon \rightarrow 0+0}(\widehat{\Phi}(\zeta+\varepsilon h)-\widehat{\Phi}(\zeta)) \varepsilon^{-1}=\widehat{\Phi}^{\prime}(\zeta) h \quad \forall h \in E_{3}\right) .
\end{gathered}
$$


$\Phi^{\prime}(\zeta)$ is the right Gateaux derivative in the point $\zeta$ and $\widehat{\Phi}^{\prime}(\zeta)$ is the left Gateaux derivative in the point $\zeta$.

A mapping $\Phi(\zeta)$ (or $\widehat{\Phi}(\zeta)$ ) of the variable $\zeta=x+y i_{2}+z i_{3} \in \Omega_{\zeta}$ with differentiable real-valued components is right- $G$-monogenic (or resp. left- $G$ monogenic) if and only if the following Cauchy-Riemann conditions are satisfied [1]:

$$
\frac{\partial \Phi}{\partial y}=i_{2} \frac{\partial \Phi}{\partial x}, \quad \frac{\partial \Phi}{\partial z}=i_{3} \frac{\partial \Phi}{\partial x}
$$

or resp.

$$
\frac{\partial \widehat{\Phi}}{\partial y}=\frac{\partial \widehat{\Phi}}{\partial x} i_{2}, \quad \frac{\partial \widehat{\Phi}}{\partial z}=\frac{\partial \widehat{\Phi}}{\partial x} i_{3} .
$$

It follows from the decomposition of the resolvent

$$
(t-\zeta)^{-1}=\frac{1}{t-\xi_{1}} e_{1}+\frac{1}{t-\xi_{2}} e_{2}, \quad \forall t \in \mathbb{C}: t \neq \xi_{1}, t \neq \xi_{2}
$$

that the points $(x, y, z) \in \mathbb{R}^{3}$ corresponding to the noninvertible elements $\zeta=x i_{1}+y i_{2}+z i_{3}$ of the algebra $\mathbb{H}(\mathbb{C})$ form the straight lines in $\mathbb{R}^{3}$ :

$$
\begin{aligned}
& L^{1}: x+y \operatorname{Re} a_{1}+z \operatorname{Re} b_{1}=0, \quad y \operatorname{Im} a_{1}+z \operatorname{Im} b_{1}=0, \\
& L^{2}: x+y \operatorname{Re} a_{2}+z \operatorname{Re} b_{2}=0, \quad y \operatorname{Im} a_{2}+z \operatorname{Im} b_{2}=0
\end{aligned}
$$

in the three-dimensional space $\mathbb{R}^{3}$.

Denote by $D_{k} \subset \mathbb{C}$ the image of $\Omega_{\zeta}$ under the mapping $f_{k}, k=1,2$. A constructive description of all right- and left- $G$-monogenic mappings by means of holomorphic functions of the complex variable are obtained in the paper [1]. Namely, proved the theorem:

Let a domain $\Omega \subset \mathbb{R}^{3}$ is convex in the direction of the straight lines $L^{1}, L^{2}$ and $f_{1}\left(E_{3}\right)=f_{2}\left(E_{3}\right)=\mathbb{C}$. Then any right- $G$-monogenic mapping $\Phi: \Omega_{\zeta} \rightarrow$ $\mathbb{H}(\mathbb{C})$ can be expressed in the form

$$
\begin{gathered}
\Phi(\zeta)=F_{1}\left(\xi_{1}\right) e_{1}+F_{2}\left(\xi_{2}\right) e_{2}+F_{3}\left(\xi_{1}\right) e_{3}+F_{4}\left(\xi_{2}\right) e_{4} \\
\forall \zeta=x i_{1}+y i_{2}+z i_{3} \in \Omega_{\zeta},
\end{gathered}
$$

where $F_{1}, F_{4}$ are the certain holomorphic in a domain $D_{1}$ functions of the variable $\xi_{1}:=x+y a_{1}+z b_{1}$ and $F_{2}, F_{3}$ are the certain holomorphic in a domain $D_{2}$ functions of the variable $\xi_{2}:=x+y a_{2}+z b_{2}$.

Under the same assumptions, any left- $G$-monogenic mapping $\widehat{\Phi}: \Omega_{\zeta} \rightarrow$ $\mathbb{H}(\mathbb{C})$ can be expressed in the form

$$
\widehat{\Phi}(\zeta)=\widehat{F}_{1}\left(\xi_{1}\right) e_{1}+\widehat{F}_{2}\left(\xi_{2}\right) e_{2}+\widehat{F}_{3}\left(\xi_{2}\right) e_{3}+\widehat{F}_{4}\left(\xi_{1}\right) e_{4},
$$


where $\widehat{F}_{n}, n=\overline{1,4}$ are defined similarly to above.

\section{Cauchy integral theorem for a curvilinear integral.}

Let $\gamma$ be a Jordan rectifiable curve in $\mathbb{R}^{3}$. For a continuous mapping $\Psi: \gamma_{\zeta} \rightarrow \mathbb{H}(\mathbb{C})$ of the form

$$
\Psi(\zeta)=\sum_{k=1}^{4} U_{k}(x, y, z) e_{k}+i \sum_{k=1}^{4} V_{k}(x, y, z) e_{k},
$$

where $(x, y, z) \in \gamma$ and $U_{k}: \gamma \rightarrow \mathbb{R}, V_{k}: \gamma \rightarrow \mathbb{R}$, we define integrals along a Jordan rectifiable curve $\gamma_{\zeta}$ by the equalities:

$$
\begin{gathered}
\int_{\gamma_{\zeta}} d \zeta \Psi(\zeta):=\sum_{k=1}^{4} e_{k} \int_{\gamma} U_{k}(x, y, z) d x+\sum_{k=1}^{4} i_{2} e_{k} \int_{\gamma} U_{k}(x, y, z) d y+ \\
+\sum_{k=1}^{4} i_{3} e_{k} \int_{\gamma} U_{k}(x, y, z) d z+i \sum_{k=1}^{4} e_{k} \int_{\gamma} V_{k}(x, y, z) d x+ \\
+i \sum_{k=1}^{4} i_{2} e_{k} \int_{\gamma} V_{k}(x, y, z) d y+i \sum_{k=1}^{4} i_{3} e_{k} \int_{\gamma} V_{k}(x, y, z) d z
\end{gathered}
$$

and

$$
\begin{aligned}
\int_{\gamma_{\zeta}} \Psi(\zeta) d \zeta:=\sum_{k=1}^{4} e_{k} \int_{\gamma} U_{k}(x, y, z) d x+\sum_{k=1}^{4} e_{k} i_{2} \int_{\gamma} U_{k}(x, y, z) d y+ \\
\quad+\sum_{k=1}^{4} e_{k} i_{3} \int_{\gamma} U_{k}(x, y, z) d z+i \sum_{k=1}^{4} e_{k} \int_{\gamma} V_{k}(x, y, z) d x+ \\
+i \sum_{k=1}^{4} e_{k} i_{2} \int_{\gamma} V_{k}(x, y, z) d y+i \sum_{k=1}^{4} e_{k} i_{3} \int_{\gamma} V_{k}(x, y, z) d z,
\end{aligned}
$$

where $d \zeta:=d x+i_{2} d y+i_{3} d z$.

Let $\Sigma$ be a piece-smooth surface in $\mathbb{R}^{3}$. For a continuous function $\Psi: \Sigma_{\zeta} \rightarrow$ $\mathbb{H}(\mathbb{C})$ of the form $(6)$, where $(x, y, z) \in \Sigma$, we define surface integrals on $\Sigma_{\zeta}$ with the differential form $\sigma:=d y d z+d z d x i_{2}+d x d y i_{3}$ by the equalities

$$
\int_{\Sigma_{\zeta}} \sigma \Psi(\zeta):=\sum_{k=1}^{4} e_{k} \int_{\Sigma} U_{k}(x, y, z) d y d z+\sum_{k=1}^{4} i_{2} e_{k} \int_{\Sigma} U_{k}(x, y, z) d z d x+
$$




$$
\begin{aligned}
& +\sum_{k=1}^{4} i_{3} e_{k} \int_{\Sigma} U_{k}(x, y, z) d x d y+i \sum_{k=1}^{4} e_{k} \int_{\Sigma} V_{k}(x, y, z) d y d z+ \\
& +i \sum_{k=1}^{4} i_{2} e_{k} \int_{\Sigma} V_{k}(x, y, z) d z d x+i \sum_{k=1}^{4} i_{3} e_{k} \int_{\Sigma} V_{k}(x, y, z) d x d y \\
& \int_{\Sigma_{\zeta}} \Psi(\zeta) \sigma:=\sum_{k=1}^{4} e_{k} \int_{\Sigma} U_{k}(x, y, z) d y d z+\sum_{k=1}^{4} e_{k} i_{2} \int_{\Sigma} U_{k}(x, y, z) d z d x+ \\
& +\sum_{k=1}^{4} e_{k} i_{3} \int_{\Sigma} U_{k}(x, y, z) d x d y+i \sum_{k=1}^{4} e_{k} \int_{\Sigma} V_{k}(x, y, z) d y d z+ \\
& +i \sum_{k=1}^{4} e_{k} i_{2} \int_{\Sigma} V_{k}(x, y, z) d z d x+i \sum_{k=1}^{4} e_{k} i_{3} \int_{\Sigma} V_{k}(x, y, z) d x d y .
\end{aligned}
$$

If a mapping $\Psi: \Omega_{\zeta} \rightarrow \mathbb{H}(\mathbb{C})$ is continuous together with partial derivatives of the first order in a domain $\Omega_{\zeta}, \Sigma$ is a piece-smooth surface in $\Omega$ and the edge $\gamma$ of surface $\Sigma$ is a rectifiable Jordan curve, then the following analogues of the Stokes formula are true:

$$
\begin{gathered}
\int_{\gamma_{\zeta}} d \zeta \Psi(\zeta)=\int_{\Sigma_{\zeta}}\left(i_{2} \frac{\partial \Psi}{\partial x}-\frac{\partial \Psi}{\partial y}\right) d x d y+\left(i_{3} \frac{\partial \Psi}{\partial y}-i_{2} \frac{\partial \Psi}{\partial z}\right) d y d z+ \\
+\left(\frac{\partial \Psi}{\partial z}-i_{3} \frac{\partial \Psi}{\partial x}\right) d z d x \\
\int_{\gamma_{\zeta}} \Psi(\zeta) d \zeta=\int_{\Sigma_{\zeta}}\left(\frac{\partial \Psi}{\partial x} i_{2}-\frac{\partial \Psi}{\partial y}\right) d x d y+\left(\frac{\partial \Psi}{\partial y} i_{3}-\frac{\partial \Psi}{\partial z} i_{2}\right) d y d z+ \\
+\left(\frac{\partial \Psi}{\partial z}-\frac{\partial \Psi}{\partial x} i_{3}\right) d z d x .
\end{gathered}
$$

Now, the next theorem is a result of the formulas (7), (8) and the equalities (2), (3), respectively.

Theorem 1. Suppose that $\Phi: \Omega_{\zeta} \rightarrow \mathbb{H}(\mathbb{C})$ is a right-G-monogenic mapping in a domain $\Omega_{\zeta}$ and $\widehat{\Phi}: \Omega_{\zeta} \rightarrow \mathbb{H}(\mathbb{C})$ is a left-G-monogenic mapping in 
$\Omega_{\zeta}$. Suppose also that $\Sigma$ is a piece-smooth surface in $\Omega$, and the edge $\gamma$ of surface $\Sigma$ is a rectifiable Jordan curve. Then

$$
\int_{\gamma_{\zeta}} d \zeta \Phi(\zeta)=\int_{\gamma_{\zeta}} \widehat{\Phi}(\zeta) d \zeta=0
$$

In the case where a domain $\Omega$ is convex, then by the usual way (see, e. g., [9]) the equality (9) can be prove for an arbitrary closed Jordan rectifiable curve $\gamma_{\zeta}$.

In the case where a domain $\Omega$ is an arbitrary, then similarly to the proof of Theorem $3.2[10]$ we can prove the following

Theorem 2. Let $\Phi: \Omega_{\zeta} \rightarrow \mathbb{H}(\mathbb{C})$ be a right-G-monogenic mapping in a domain $\Omega_{\zeta}$ and $\widehat{\Phi}: \Omega_{\zeta} \rightarrow \mathbb{H}(\mathbb{C})$ be a left-G-monogenic mapping in $\Omega_{\zeta}$. Then for every closed Jordan rectifiable curve $\gamma_{\zeta}$ homotopic to a point in $\Omega_{\zeta}$, the the equalities (9) holds.

\section{Cauchy integral formula.}

To establish the Cauchy integral formula for a curvilinear integral, consider the following auxiliary statement.

Lemma 1. Suppose that a domain $\Omega \subset \mathbb{R}^{3}$ is convex in the direction of the straight lines $L^{1}, L^{2}$ and $f_{1}\left(E_{3}\right)=f_{2}\left(E_{3}\right)=\mathbb{C}$. Suppose also that $\Phi: \Omega_{\zeta} \rightarrow \mathbb{H}(\mathbb{C})$ is a right-G-monogenic mapping in $\Omega_{\zeta}$, and $\widehat{\Phi}: \Omega_{\zeta} \rightarrow \mathbb{H}(\mathbb{C})$ is a left-G-monogenic mapping in $\Omega_{\zeta}$, and $\gamma_{\zeta}$ is an arbitrary rectifiable curve in $\Omega_{\zeta}$. Then

$$
\int_{\gamma_{\zeta}} d \zeta \Phi(\zeta)=e_{1} \int_{\gamma_{1}} F_{1}\left(\xi_{1}\right) d \xi_{1}+e_{2} \int_{\gamma_{2}} F_{2}\left(\xi_{2}\right) d \xi_{2}+e_{3} \int_{\gamma_{1}} F_{3}\left(\xi_{1}\right) d \xi_{1}+e_{4} \int_{\gamma_{2}} F_{4}\left(\xi_{2}\right) d \xi_{2},
$$

and respectively

$$
\int_{\gamma_{\zeta}} \widehat{\Phi}(\zeta) d \zeta=e_{1} \int_{\gamma_{1}} \widehat{F}_{1}\left(\xi_{1}\right) d \xi_{1}+e_{2} \int_{\gamma_{2}} \widehat{F}_{2}\left(\xi_{2}\right) d \xi_{2}+e_{3} \int_{\gamma_{2}} \widehat{F}_{3}\left(\xi_{2}\right) d \xi_{2}+e_{4} \int_{\gamma_{1}} \widehat{F}_{4}\left(\xi_{1}\right) d \xi_{1},
$$

where $\gamma_{1}, \gamma_{2}$ are the images of $\gamma_{\zeta}$ under the mappings $f_{1}, f_{2}$ and $F_{n}, \widehat{F}_{n}, n=$ $\overline{1,4}$ are the same functions as in (4) and (5) respectively.

Proof. The equality (10) follows immediately from the representation (4), the equality $d \zeta=d \xi_{1} e_{1}+d \xi_{2} e_{2}$ and the multiplication rules (1). Similarly we can prove the equality (11). The Lemma is proved.

Let $\zeta \in E_{3}$. An inverse element $\zeta^{-1}$ is of the following form:

$$
\zeta^{-1}=\frac{1}{\xi_{1}} e_{1}+\frac{1}{\xi_{2}} e_{2}
$$


and it exists if and only if $\xi_{1} \neq 0$ and $\xi_{2} \neq 0$.

Let $\zeta_{0}=\xi_{1}^{(0)} e_{1}+\xi_{2}^{(0)} e_{2}$ be a point in a domain $\Omega_{\zeta} \subset E_{3}$. In a neighborhood of $\zeta_{0}$ contained in $\Omega_{\zeta}$ let us take a circle $C\left(\zeta_{0}\right)$ with the center at the point $\zeta_{0}$. By $C_{k} \subset \mathbb{C}$ we denote the image of $C\left(\zeta_{0}\right)$ under the mapping $f_{k}, k=1,2$. We assume that the circle $C\left(\zeta_{0}\right)$ embraces the set $\left\{\zeta-\zeta_{0}: \zeta \in L_{\zeta}^{1} \cup L_{\zeta}^{2}\right\}$. It means that $C_{k}$ bounds some domain $D_{k}^{\prime}$ and $\xi_{k}^{(0)} \in D_{k}^{\prime}, \quad k=1,2$.

We say that the curve $\gamma_{\zeta} \subset \Omega_{\zeta}$ embraces once the set $\left\{\zeta-\zeta_{0}: \zeta \in\right.$ $\left.L_{\zeta}^{1} \cup L_{\zeta}^{2}\right\}$, if there exists a circle $C\left(\zeta_{0}\right)$ which embraces the mentioned set and is homotopic to $\gamma_{\zeta}$ in the domain $\Omega_{\zeta} \backslash\left\{\zeta-\zeta_{0}: \zeta \in L_{\zeta}^{1} \cup L_{\zeta}^{2}\right\}$.

Theorem 3. Suppose that a domain $\Omega \subset \mathbb{R}^{3}$ is convex in the direction of the straight lines $L^{1}, L^{2}$ and $f_{1}\left(E_{3}\right)=f_{2}\left(E_{3}\right)=\mathbb{C}$. Suppose also that $\Phi: \Omega_{\zeta} \rightarrow \mathbb{H}(\mathbb{C})$ is a right-G-monogenic mapping in $\Omega_{\zeta}$ and $\widehat{\Phi}: \Omega_{\zeta} \rightarrow \mathbb{H}(\mathbb{C})$ is a left-G-monogenic mapping in $\Omega_{\zeta}$. Then for every point $\zeta_{0} \in \Omega_{\zeta}$ the following equalities are true:

$$
\Phi\left(\zeta_{0}\right)=\frac{1}{2 \pi i} \int_{\gamma_{\zeta}}\left(\zeta-\zeta_{0}\right)^{-1} d \zeta \Phi(\zeta)
$$

and

$$
\widehat{\Phi}\left(\zeta_{0}\right)=\frac{1}{2 \pi i} \int_{\gamma_{\zeta}} \widehat{\Phi}(\zeta)\left(\zeta-\zeta_{0}\right)^{-1} d \zeta
$$

where $\gamma_{\zeta}$ is an arbitrary closed Jordan rectifiable curve in $\Omega_{\zeta}$, that embraces once the set $\left\{\zeta-\zeta_{0}: \zeta \in L_{\zeta}^{1} \cup L_{\zeta}^{2}\right\}$.

Proof. Inasmuch as $\gamma_{\zeta}$ is homotopic to $C\left(\zeta_{0}\right)$ in the domain $\Omega_{\zeta} \backslash\left\{\zeta-\zeta_{0}\right.$ : $\left.\zeta \in L_{\zeta}^{1} \cup L_{\zeta}^{2}\right\}$, it follows from Theorem 2 that

$$
\frac{1}{2 \pi i} \int_{\gamma_{\zeta}}\left(\zeta-\zeta_{0}\right)^{-1} d \zeta \Phi(\zeta)=\frac{1}{2 \pi i} \int_{C\left(\zeta_{0}\right)}\left(\zeta-\zeta_{0}\right)^{-1} d \zeta \Phi(\zeta)
$$

Further, using the equality (12), Lemma 1 and the integral Cauchy formula for holomorphic functions $F_{n}, n=\overline{1,4}$, we obtain immediately the following equalities:

$$
\begin{gathered}
\frac{1}{2 \pi i} \int_{C\left(\zeta_{0}\right)}\left(\zeta-\zeta_{0}\right)^{-1} d \zeta \Phi(\zeta)=e_{1} \frac{1}{2 \pi i} \int_{C_{1}} \frac{F_{1}\left(\xi_{1}\right)}{\xi_{1}-\xi_{1}^{(0)}} d \xi_{1}+e_{2} \frac{1}{2 \pi i} \int_{C_{2}} \frac{F_{2}\left(\xi_{2}\right)}{\xi_{2}-\xi_{2}^{(0)}} d \xi_{2}+ \\
+e_{3} \frac{1}{2 \pi i} \int_{C_{1}} \frac{F_{3}\left(\xi_{1}\right)}{\xi_{1}-\xi_{1}^{(0)}} d \xi_{1}+e_{4} \frac{1}{2 \pi i} \int_{C_{2}} \frac{F_{4}\left(\xi_{2}\right)}{\xi_{2}-\xi_{2}^{(0)}} d \xi_{2}=
\end{gathered}
$$




$$
=F_{1}\left(\xi_{1}^{(0)}\right) e_{1}+F_{2}\left(\xi_{2}^{(0)}\right) e_{2}+F_{3}\left(\xi_{1}^{(0)}\right) e_{3}+F_{4}\left(\xi_{2}^{(0)}\right) e_{4}=\Phi\left(\zeta_{0}\right),
$$

where $\zeta_{0}=\xi_{1}^{(0)} e_{1}+\xi_{2}^{(0)} e_{2}$. Similarly can be proved the equality (14). The Theorem is proved.

We note that the method of this proof is similarly to the proof of the Theorem 6 of the paper [8], where Cauchy integral formula is obtained in a finite-dimensional semi-simple commutative algebra.

\section{Cauchy integral theorem for a surface integral.}

Let $\Omega$ be a bounded domain in $\mathbb{R}^{3}$. For a continuous mapping $\Psi: \Omega_{\zeta} \rightarrow$ $\mathbb{H}(\mathbb{C})$ of the form $(6)$, where $(x, y, z) \in \Omega$ and $U_{k}: \Omega \rightarrow \mathbb{R}, V_{k}: \Omega \rightarrow \mathbb{R}$, we define a volume integral by the equality

$$
\int_{\Omega_{\zeta}} \Psi(\zeta) d x d y d z:=\sum_{k=1}^{4} e_{k} \int_{\Omega} U_{k}(x, y, z) d x d y d z+i \sum_{k=1}^{4} e_{k} \int_{\Omega} V_{k}(x, y, z) d x d y d z
$$

Let $\Sigma$ be a piece-smooth surface in $\mathbb{R}^{3}$. For a continuous mapping $\Psi: \Sigma_{\zeta} \rightarrow$ $\mathbb{H}(\mathbb{C})$ of the form $(6)$, where $(x, y, z) \in \Sigma$ and $U_{k}: \Sigma \rightarrow \mathbb{R}, V_{k}: \Sigma \rightarrow \mathbb{R}$, we define the surface integrals on a piece-smooth surface $\Sigma_{\zeta}$ with the differential form $\sigma:=d y d z+d z d x i_{2}+d x d y i_{3}$ by the equalities

$$
\begin{aligned}
\int_{\Sigma_{\zeta}} \sigma & \Psi(\zeta):=\sum_{k=1}^{4} e_{k} \int_{\Sigma} U_{k}(x, y, z) d y d z+\sum_{k=1}^{4} i_{2} e_{k} \int_{\Sigma} U_{k}(x, y, z) d z d x+ \\
& +\sum_{k=1}^{4} i_{3} e_{k} \int_{\Sigma} U_{k}(x, y, z) d x d y+i \sum_{k=1}^{4} e_{k} \int_{\Sigma} V_{k}(x, y, z) d y d z+ \\
& +i \sum_{k=1}^{4} i_{2} e_{k} \int_{\Sigma} V_{k}(x, y, z) d z d x+i \sum_{k=1}^{4} i_{3} e_{k} \int_{\Sigma} V_{k}(x, y, z) d x d y
\end{aligned}
$$

and

$$
\begin{aligned}
& \int_{\Sigma_{\zeta}} \Psi(\zeta) \sigma:=\sum_{k=1}^{4} e_{k} \int_{\Sigma} U_{k}(x, y, z) d y d z+\sum_{k=1}^{4} e_{k} i_{2} \int_{\Sigma} U_{k}(x, y, z) d z d x+ \\
& \quad+\sum_{k=1}^{4} e_{k} i_{3} \int_{\Sigma} U_{k}(x, y, z) d x d y+i \sum_{k=1}^{4} e_{k} \int_{\Sigma} V_{k}(x, y, z) d y d z+ \\
& \quad+i \sum_{k=1}^{4} e_{k} i_{2} \int_{\Sigma} V_{k}(x, y, z) d z d x+i \sum_{k=1}^{4} e_{k} i_{3} \int_{\Sigma} V_{k}(x, y, z) d x d y .
\end{aligned}
$$


If a domain $\Omega \subset \mathbb{R}^{3}$ has a closed piece-smooth boundary $\partial \Omega$ and a mapping $\Psi: \Omega_{\zeta} \rightarrow \mathbb{H}(\mathbb{C})$ is continuous together with partial derivatives of the first order up to the boundary $\partial \Omega_{\zeta}$, then the following analogues of the Gauss Ostrogradsky formula are true:

$$
\int_{\partial \Omega_{\zeta}} \sigma \Psi(\zeta)=\int_{\Omega_{\zeta}}\left(\frac{\partial \Psi}{\partial x}+i_{2} \frac{\partial \Psi}{\partial y}+i_{3} \frac{\partial \Psi}{\partial z}\right) d x d y d z .
$$

and

$$
\int_{\partial \Omega_{\zeta}} \Psi(\zeta) \sigma=\int_{\Omega_{\zeta}}\left(\frac{\partial \Psi}{\partial x}+\frac{\partial \Psi}{\partial y} i_{2}+\frac{\partial \Psi}{\partial z} i_{3}\right) d x d y d z .
$$

Now, the next theorem is a result of the formulas (15), (16) and the conditions (2), (3), respectively.

Theorem 4. Suppose that $\Omega$ has a closed piece-smooth boundary $\partial \Omega$. Suppose also that the mapping $\Phi: \Omega_{\zeta} \rightarrow \mathbb{H}(\mathbb{C})$ is a right-G-monogenic in $\Omega_{\zeta}$ and $\widehat{\Phi}: \Omega_{\zeta} \rightarrow \mathbb{H}(\mathbb{C})$ is a left-G-monogenic in $\Omega_{\zeta}$, and these mappings are continuous together with partial derivatives of the first order up to the boundary $\partial \Omega_{\zeta}$. Then

$$
\int_{\partial \Omega_{\zeta}} \sigma \Phi(\zeta)=\int_{\Omega_{\zeta}}\left(1+i_{2}^{2}+i_{3}^{2}\right) \Phi^{\prime}(\zeta) d x d y d z
$$

and

$$
\int_{\partial \Omega_{\zeta}} \widehat{\Phi}(\zeta) \sigma=\int_{\Omega_{\zeta}} \widehat{\Phi}^{\prime}(\zeta)\left(1+i_{2}^{2}+i_{3}^{2}\right) d x d y d z .
$$

Corollary. Under the conditions of Theorem 4 with the additional assumption $1+i_{2}^{2}+i_{3}^{2}=0$, i. e. the mappings $\Phi$ and $\widehat{\Phi}$ are solutions of the three-dimensional Laplace equation, then the equalities (17) and (18) can be rewritten in the form

$$
\int_{\partial \Omega_{\zeta}} \sigma \Phi(\zeta)=\int_{\partial \Omega_{\zeta}} \widehat{\Phi}(\zeta) \sigma=0
$$

\section{References}

[1] V.S. Shpakivskyi and T.S. Kuzmenko, On one class of quaternionic mappings, Ukr. Math. J. 68 (1) (2016) 117-130.

[2] G. C. Moisil and N. Theodoresco, Functions holomorphes dans l'espace, Mathematica (Cluj), 5 (1931), 142 - 159. 
[3] R. Fueter, Die Funktionentheorie der Differentialgleichungen $\Delta u=0$ und $\Delta \Delta u=0$ mit vier reellen Variablen, Comment. math. helv. 7 (1935), $307-330$.

[4] B. Schuler, Zur Theorie der regulären Funktionen einer QuaternionenVariablen, Comment. math. Helv. 10 (1937), 327 - 342.

[5] A. Sudbery, Quaternionic analysis, Math. Proc. Camb. Phil. Soc., 85 (1979), $199-225$.

[6] O.F. Herus, On hyperholomorphic functions of the space variable, Ukr. Math. J. 63 (4) (2011), $530-537$.

[7] G. Gentili, C. Stoppato and D. Struppa, Regular Functions of a Quaternionic Variable, Springer Monographs in Mathematics, 2013.

[8] S. A. Plaksa and R.P. Pukhtaevich, Constructive description of monogenic functions in n-dimensional semi-simple algebra, An. Şt. Univ. Ovidius Constanţa, 22 (1) (2014), 221 - 235.

[9] I. I. Privalov, Introduction to the Theory of Functions of a Complex Variable, GITTL, Moscow, 1977. [in Russian]

[10] E. K. Blum, A theory of analytic functions in banach algebras, Trans. Amer. Math. Soc., 78 (1955), 343 - 370.

Vitalii SHPAKIVSKYI,

Department of complex analysis and potential theory,

Institute of Mathematics of the National Academy of Science of Ukraine,

3 Tereshchenkivs'ka Street, Kyiv, Ukraine.

Email: shpakivskyi86@gmail.com, shpakivskyi@imath.kiev.ua

Tetyana KUZMENKO,

Department of complex analysis and potential theory,

Institute of Mathematics of the National Academy of Science of Ukraine,

3 Tereshchenkivs'ka Street, Kyiv, Ukraine.

Email: kuzmenko.ts15@gmail.com 